\title{
ALTERNATIVAS DE SALUD EN EL TERCER MUNDO
}

\author{
E.R. Weiss-Altaner \\ El Colegio de México
}

\begin{abstract}
AUNQUE El RITMO que ha cobrado el descenso de la mortalidad y morbilidad ( también llamado "transición en mortalidad") en los países menos desarrollados en los últimos cuatro decenios, es mayor que la velocidad con que cayeron la mortalidad y la morbilidad en los países más desarrollados, todavía persisten graves diferencias entre ambos grupos de países. La esperanza de vida al nacer en los países más desarrollados aumentó en $25 \%$ en treinta años, desde un nivel de 56 años en 1935-1939 a un nivel de 70 años en 1965-1970.' En el mismo intervalo, la esperanza de vida al nacer en los países menos desarrollados (en Asia meridional y oriental, Africa y América Latina) creció en 53\%, desde 32 a 49 años." Además, el acercamiento en la esperanza de vida de ambos grupos de países ha sido mucho más marcado en las edades adultas, lo que señala las agudas diferencias que aún existen en la mortalidad infantil según el nivel económico alcanzado por una nación. Finalmente, en los países menos desarrollados, las aflicciones infecciosas, parasitarias y respiratorias, y las enfermedades del aparato digestivo, cobran una importancia superada ya en las economías más avanzadas, donde cánceres y padecimientos circulatorios ocupan ese lugar de importancia.

¿Cómo explicar las trayectorias que han seguido la mortalidad y la morbilidad en distintos países? Un conocimiento del mecanismo social en que se producen los fenómenos de mortalidad y morbilidad permitirá responder a esta pregunta, así como enumerar las posibilidades en cuanto a políticas de salud entre las que debe escoger la colectividad, especialmente por medio del aparato estatal.
\end{abstract}

\section{CREAción de SAlud}

La salud es algo producido. Esta idea es fundamental para entender el mecanismo en que surgen la mortalidad y la morbilidad. Mediante la combinación de insumos - como ejercicio, dieta, vivienda, tratamiento mé-

\footnotetext{
${ }^{1}$ Banco Mundial, Population Policies and Economic Development, Baltimore y Londres, John Hopkins University Press, 1974, cuadro anexo suplementario Núm. 2.

Ibid.
} 
dico, y, en particular, el tiempo que ella dedica a distintas actividades-, la persona produce su salud. Importan no sólo el número de horas que la persona gasta en distintas actividades, sino también las características concretas de las actividades mismas: el trabajo de oficinista no afecta la salud de la misma manera que extraer carbón.

La salud, expresada en términos de días sin aflicción fisiológica o mental, tiene una doble dimensión económica: es un objeto de consumo, en tanto que padecimientos fisiológicos y mentales ocasionan desutilidad o disgusto al individuo; y es un objeto de inversión, porque el grado de salud determina el tiempo total que la persona puede dedicar a la producción de bienes, tanto para uso propio como para vender. Los bienes que no se fabricaron, por razón de la muerte o enfermedad del trabajador -en virtud de su ausencia total del trabajo o solamente de la reducción de su productividad normal-, miden el rendimiento material de los gastos hechos en mejorar y mantener la salud del trabajador, considerados estos gastos como una inversión en el trabajador. Mirar a la salud como algo producido permite hablar de un "monto de salud" que sufre desgaste, pero que puede ser aumentado en virtud de la inversión recién mencionada. La tasa de depreciación del monto de salud personal varía acorde con la edad del individuo, y seguramente el perfil por edades de la tasa de depreciación cambiará según la etapa histórica y el grupo social en que se encuentra la persona. En otras palabras, la salud personal experimenta un ritmo de desgaste menor, a cualquier edad, ahora que hace un siglo; mientras que, en toda época y a cualquier edad, la salud de una persona acomodada disminuye más lentamente que la salud de alguien con medios económicos muy limitados.

Según el análisis económico, la cantidad de salud observada —reflejada en la estadística de esperanza de vida y de morbilidad- sería el resultado de la interacción entre la cantidad de salud que una persona desea ("demanda de salud") y la cantidad que ella puede producir ("oferta de salud"). El saldo entre estas magnitudes hipotéticas puede caer en tres zonas:

1) equilibrio: demanda y oferta cobran valores muy cercanos;

2) demanda insatisfecha: la demanda de salud es muy alta, o la oferta es insuficiente;

3 ) oferta en exceso: la persona está produciendo menos salud de la que es capaz, en virtud de una demanda muy alta de bienes que merman la salud.

Como se verá a continuación, diferencias en los niveles que cobren los determinantes de la demanda y oferta personales de salud hacen muy probable que diferentes grupos de la población registren, en un período dado, distintos saldos entre su oferta y demanda de salud, de modo que los grupos más pobres vivan con una demanda insatisfecha, mientras que los grupos más adinerados experimenten una oferta en exceso. Asimismo, una persona puede pasar por los diversos saldos de salud en el transcurso de su vida, porque su ingreso y condiciones de trabajo iniciales quizá no le permitan producir la magnitud de salud que desea, mientras que la salud 
que podría producir, en atención solamente a su ingreso acrecentado, quizá más adelante en su vida rebase la salud que de hecho produce, en virtud de la composición del consumo y del tren de vida que le brindarán y exigirán entonces su mayor ingreso y posición social.

\section{Demanda de SAlud}

Igual como sucede con otros objetos de consumo e inversión, la cantidad de salud que alguien desea obedece, en primer lugar: al goce subjetivo que le proporciona la salud en comparación con otras fuentes de satisfacción, al costo relativo de "conseguir" (o sea, de producir) salud y al poder adquisitivo de la persona (que representa su capacidad de apropiar productos del trabajo). El poder adquisitivo actual mide la capacidad actual de apropiar productos del trabajo, entre los cuales están los insumos usados en crear salud. El poder adquisitivo vitalicio esperado por la persona le indica su capacidad de apropiación a lo largo de su vida, y también le da una idea del rédito absoluto que puede esperar al invertir parte de su ingreso en alargar su vida y en amenguar la frecuencia y virulencia de las enfermedades.

El impacto parcial sobre la cantidad de salud deseada por la persona sería: positivo en cuanto al ingreso actual y al vitalicio; negativo en cuanto al costo relativo de la salud; y positivo en cuanto a la satisfacción subjetiva proporcionada por la salud en comparación con otras fuentes de satisfacción. Como existen bienes que merman la salud pero son altamente deseados por sus otras virtudes, y que compiten por un lugar en "la canasta de consumo deseada" con las actividades y bienes que aumentan y mantienen la salud, es posible que un cambio en el ingreso personal disminuya la cantidad deseada de salud en vez de aumentarla, por crecer más rápidamente la demanda de los sustitutivos de la buena salud que la demanda de bienes que la mantienen y mejoran. Sucede así con drogas como alcohol y tabaco, así como con el consumo excesivo de grasas y carbohidratos. Entonces cabe también esperar, quizá con un retraso de varios años, más gasto en tratamiento médico y en otros medios para contrarrestar el aumento en la tasa de desgaste de la salud y el descenso en el monto inicial de salud debidos a la composición insalubre del consumo.

Por cierto, ninguno de los determinantes directos de la cantidad de salud deseada por el individuo está desligado del más amplio contexto social en que éste se ubica. La magnitud del ingreso personal y su perfil a lo largo de la vida del trabajador se ven determinados por, entre otros, el desarrollo tecnológico y la contienda entre grupos sociales tanto del país como extranjeros. Tensiones inherentes en el tren de vida que debe llevar la gente fomentan el consumo de muchos bienes insalubres, cuyo impacto nocivo es opacado por una publicidad sutil que busca fortalecer la demanda de esos bienes por vía de los gustos individuales. 


\section{OfERTA DE SAlud}

Falta la cantidad de salud que la persona puede producir — cantidad que merece el nombre de "oferta de salud"- para completar el mecanismo que genera la salud observada en las estadísticas correspondientes. Antes de pasar revista a los determinantes inmediatos de la oferta individual de salud, es importante notar el vínculo entre esta última y el uso que la persona hace de su tiempo, en virtud de que diferentes actividades tienen un diferente impacto unitario sobre la salud individual. Además, los factores que determinan el uso del tiempo influyen también en algunos aspectos de la oferta de salud (como se verá más adelante), lo que señala un enlace teórico dentro del pensamiento demográfico, entre la mortalidad y la participación en la vida activa.

La asignación de su tiempo que hace alguien con libertad de decisión al respecto, depende de la cantidad que desee tener de los frutos de diversas actividades (la cual a su vez es resultado de la interacción entre el ingreso personal, la estructura de precios y costos, y la estructura de preferencias subjetivas en cuanto a diversos bienes); del gusto subjetivo que la persona deriva de participar en las diferentes actividades; y de su eficiencia productiva, que indica el tiempo necesario por unidad del producto en cuestión. Por ejemplo, si se excluye la apropiación de bienes por la fuerza, la demanda de bienes vendidos en el mercado sólo puede ser cubierta con dinero, lo que impulsa a la persona a conseguir un ingreso monetario, notablemente vendiendo la fuerza de trabajo y los productos del trabajo suyos y de sus familiares. Igualmente, para satisfacer la demanda de bienes domésticos, hay que dedicar tiempo al hogar. Actividades domésticas de por sí pueden originar satisfacción subjetiva y atraer tiempo hacia sí; tales gustos, cuidadosamente inculcados en la población, contribuyen a mantener la especialización femenina en tareas del hogar.

La asignación del tiempo, entre tareas dentro y fuera del hogar, entonces, contribuye a determinar la oferta individual de salud, en virtud de las cuatro fuentes que alimentan esa oferta: la participación directa de la persońa en cuestión en actividades de consumo y producción dentro y fuera de su hogar, más la repercusión sobre su salud de las actividades de hogares y de unidades no hogareñas en las que no interviene la persona en cuestión (cuadro 1). Esta clasificación destaca la dimensión sociológica oculta en el enunciado sobre el desigual impacto sobre la salud que cabe esperarse de distintas actividades. Hogares y unidades no hogareñas tienen diferentes objetivos y estructuras de gestión, se especializan en bienes distintos y producen con técnicas y a escalas diferentes, y la impor-. tancia de sus consecuencias indirectas en materia de salud no suelen ser las mismas. Es de cardinal importancia subrayar que, si bien las personas libres deciden sobre el uso de su tiempo, tomando parte en actividades que impactan su salud de manera desigual, ellas normalmente no conservan el poder de gestión más que en su propio hogar, y por consiguiente no pueden controlar las repercusiones salubres o insalubres de todas las 


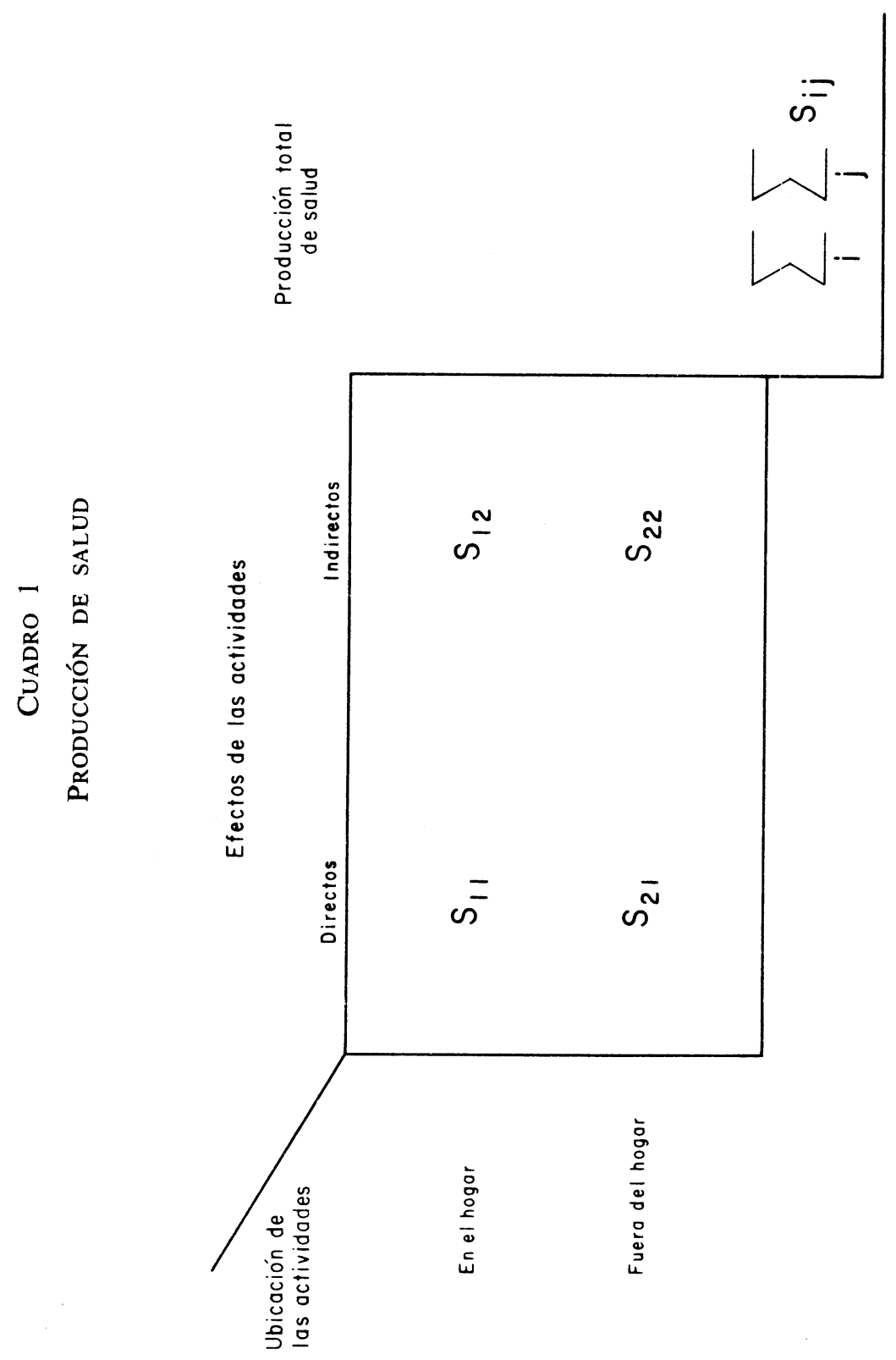


tareas entre las que han repartido su tiempo, y menos controlar las repercusiones de las actividades en las que no intervienen directamente.

En el hogar, el poder adquisitivo, los precios y costos de los insumos, y las preferencias subjetivas en cuanto a insumos, productos y las actividades mismas, se unen a la eficacia productiva de los miembros del hogar para determinar, en primera instancia, la cantidad de salud que pueden aportar las actividades hogareñas tanto a los miembros del hogar que en ellas participan, como a terceros por la vía indirecta recién mencionada. Es obvio que diferencias entre hogares en cuanto a los factores indicados, particularmente en el ingreso y en sus conocimientos sobre higiene y medicina, son la herencia del reparto de poder que rige en la sociedad.

En las unidades no hogareñas (como talleres, fábricas y plantaciones), las consecuencias de sus actividades sobre la salud de los que ahí trabajan, así como sobre terceros no participantes, normalmente revisten una importancia secundaria entre los objet „vos de la empresa, en comparación con la sobrevivencia y la expansión de ésta. Aunque la creciente tecnificación de muchas tareas productivas - que exige menor ausentismo y operarios más despiertos y preparados- acentúa el interés de la empresa en condiciones de trabajo más salubres, no acalla las sospechas de que son presiones políticas - de parte de los trabajadores de las empresas y del público de terceros cuya salud siente el efecto de las actividades empresariales- las que más pesan para que las unidades no hogareñas canalicen recursos propios hacia la eliminación de las consecuencias insalubres, directas e indirectas, de sus actividades. Como está en juego parte de los beneficios que captan las unidades no hogareñas, éstas, muy racionalmente, buscan eludir la responsabilidad económica por el impacto insalubre de sus actividades, tratando de transferir los costos de las mejoras necesarias a los trabajadores directamente involucrados o a la colectividad en general.

\section{TRANSICIÓN EN MORTALIDAD}

La estructura hipotética impuesta aquí a la producción de salud, una vez cotejada con la etiología conocida de diversas causas de muerte, ayuda a comprender por qué, en un período determinado, se debilitaron éstas causas de muerte y no aquéllas, y también apunta a la política de salud por seguir para combatir las aflicciones que perduran. Algunas enfermedades reciben impulso de los quehaceres domésticos, mientras que otras sienten más la actividad fuera del hogar, en tanto que aún otras cobran fuerza de los efectos indirectos del trabajo hogareño y extrahogareño.

Al respecto, resulta instructivo considerar las enfermedades infecciosas, parasitarias y respiratorias, cuyo descenso provocó gran parte del aumento en la esperanza de vida en los países más desarrollados, mientras que todavía causan una apreciable mortalidad y morbilidad en los países me- 
nos desarrollados. Estas enfermedades son muy sensibles al "costo de transporte" que afrontan sus agentes patógenos y vectores de enfermedad, y los cuatro componentes de la oferta de salud afectan ese "costo de transporte" de manera desigual. Por lo tanto, al descubrir cómo afectaron a los cuatro componentes de la oferta de salud los cambios ocurridos en la sociedad durante el descenso en la frecuencia de esas enfermedades, la investigación revelará precisamente cómo y cuáles de esos cambios sociales produjeron el descenso observado. Paralelamente, al demostrar cuáles de los cuatro componentes fueron menos tocados por los cambios sociales, podrá vislumbrarse el sendero que debe seguir la política de salud (y su política económica adjunta, que siempre la acompaña, oculta o explícitamente) para lograr que se prolongue el descenso ya iniciado.

Dividamos las enfermedades infecciosas, parasitarias y respiratorias según el "medio de transporte" que utilizan: excremento, aire, vectores de enfermedad, o el contacto directo. ${ }^{3}$ En un barrio de viviendas densamente habitadas y con poca luz y ventilación, las enfermedades transmitidas por el aire y el contacto directo encuentran una "red de transporte" rápida y "barata" (lo mismo vale en unidades no hogareñas como talleres y fábricas). Un aumento en la disponibilidad de viviendas por habitante, por lo tanto, debería perfilar un aumento en los "costos de transporte" de esas enfermedades y una consiguiente reducción en su frecuencia; efectivamente, tales mejoras guardan relación directa con la reducción en la mortalidad por enfermedades infecciosas en los países ahora desarrollados. Los insumos utilizados en el trabajo hogareño y no hogareño, especialmente el agua y los servicios de sanidad, pueden facilitar, si son de baja calidad, la difusión de enfermedades transmitidas por vía de excrementos. Son de especial interés los insumos que contribuyen a la nutrición del hogar, porque ésta influye en la resistencia del organismo a la enfermedad y, quizás de mayor importancia, en su motivación para y capacidad de aprender. La calidad y cantidad del consumo hogareño, así como los conocimientos de los miembros del hogar sobre higiene, alimentación y salud en general, están estrechamente ligados con el nivel de ingreso del hogar. Hoy en día se debate entre historiadores de la población acerca de la importancia de las mejoras en el conocimiento médico, relativas al crecimiento en el ingreso por persona (indicador aproximado éste de mejoría en las condiciones de vida personales), en tanto causas de la transición en

3 En América Latina en el decenio de 1969, las enfermedades transmitidas por vía del excremento ( $V . g c$. , parásitos intestinales, diarreas infecciosas, poliomelitis, tifoidea, cólera) y por vía del aire ( $V . g c$., tuberculosis, pulmonía, difteria, bronquitis, tos convulsiva, meningitis, influenza, sarampión, viruela, varicela) constituyeron una fracción importante de la mortalidad total, y en especial de la mortalidad en el grupo etario 0-5. Véase: Ruth R. Puffer y Carlos V. Serrano, Inter-American Investigation of Mortality in Childhood. Provisional Report. Organización Panamericana de la Salud. Washington, D.C., septiembre de 1971. Véase también: Ruth R. Puffer y G. Wayne Griffith, Patterns of Urban Mortality. Publicación Científica No. 151. Organización Panamericana de la Salud. Washington, D.C., septiembre de 1967. 
mortalidad. La polémica se encuentra todavía en la etapa de acumular apreciaciones cuidadosas de situaciones locales, pero muchas de éstas atestiguan que los aumentos en el poder adquisitivo personal corren parejos con cambios salubres en la composición del consumo.

Los efectos indirectos de las actividades hogareñas y no hogareñas cobran especial importancia en la difusión de las enfermedades infecciosas y parasitarias, principalmente en relación a la manera de disponer de los desechos y desperdicios de esas actividades. Es aquí, en el renglón de las consecuencias insalubres indirectas de los hogares, donde los esfuerzos en salud pública $-V . g c$., suministro de agua potable, drenaje, combate contra mosquitos y otros vectores de enfermedad - han logrado su contribución sustancial a la transición en mortalidad, en el siglo pasado como en el actual. Además, los efectos indirectos negativos sobre la salud incentivan la intervención colectiva y la creación de actividades de salud pública, como se verá a continuación.

\section{SAlud pública}

Los efectos indirectos insalubres representan costos privados transferidos a terceros; existen porque la situación económica de la unidad de origen (hogareña y no hogareña) no le permite sufragar el costo en cuestión, o porque la constelación de poder en la sociedad le permite socializar parte de sus costos de producción bajo la forma de daños a la salud de terceros. La existencia de apreciables efectos indirectos negativos sobre la salud incentiva algún tipo de acción colectiva, tanto para que quienes los originan corran con parte de los costos de eliminarlos, como para asegurar que los terceros beneficiados por las eventuales mejoras también ayuden a cubrir esos costos. En ausencia de cualquier intervención colectiva y de fuertes sentimientos de solidaridad social, es difícil concebir que el egoísmo impulsara a hogares y a unidades no hogareñas a neutralizar, por cuenta propia, sus efectos insalubres sobre terceros. Estos efectos no son una mercancía y no existe un mercado en que la demanda de su eliminación (que tendrían los terceros afectados) pueda encontrarse con la oferta de su eliminación (que brindarían los que crean esos efectos). Dentro de la sociabilidad que caracteriza a la producción de mercancías, no existe una manera fácil y barata de calcular el precio de eliminar los insalubres efectos indirectos ni de cobrarlo a los múltiples beneficiarios. ¿Por qué los que originan esos efectos habrían de absorber todo el costo de eliminación, cuando los terceros beneficiados no se verían obligados, por las reglas de la circulación de mercancías, a pagar su parte correspondiente (igual al incremento descontado de su ingreso vitalacio consiguiente a la mejoría de su salud que seguiría a la eliminación de los efectos negativos indirectos en cuestión)? Además, la tecnología más eficiente para eliminar muchas externalidades negativas en materia de salud - $V . g c$., saneamiento del agua y drenaje-, así como el riesgo asociado 
con tales proyectos, pueden rebasar la capacidad económica de cualquier grupo privado que pensara lanzar esos servicios al mercado. En el caso de la contaminación del aire, el saneamiento es costoso y su distribución como mercancía ímposible, salvo mediante un recurso de ciencia ficción como la venta de máscaras anticontaminantes. No sucede así con el agua, que fácilmente pueda transformarse en mercancía; en este caso, la socialización y estatización del suministro de agua potable obedece a que el saneamiento y distribución del agua encierra apreciables economías de escala, lo que impulsa la construcción de grandes instalacicnes que absorben muchos fondos en su construcción y manutención.

La centralización, en burocracias de salud estatales y privadas, del poder de gestión sobre fondos asignados a programas de salud, no obedece principalmente a los determinantes técnicos que dan acta de nacimiento a la intervención colectiva en la salud -V.gc., las economías de escala, indivisibilidades y riesgos asociados con la eliminación de los efectos indirectos negativos sobre la salud-, sino a criterios políticos. Esa centralización, en tanto enajenación del poder de la mayoría de la población sobre la recaudación y uso de los fondos sociales para la salud es a la vez resultado e instrumento de reproducción de relaciones de producción explotativas y enajenantes.

El estado, igual que el individuo, desea salud por el goce subjetivo y no solamente por el rédito material que ella proporciona. Así se puede interpretar los a veces amplios gastos fiscales en deportes, en aras del renombre patrio. Por lo demás, la ideología de "servicio al pueblo", si de veras orienta la acción estatal y no es una mera patraña, equivale a una alta utilidad subjetiva de parte del aparato estatal por la salud y otras dimensiones del bienestar de la población. Sin embargo, parecen ser motivos inversionistas los que más animan las erogaciones fiscales en salud: aumentar el tiempo de trabajo disponible y su calidad, así como coartar el derroche de recursos pretéritos y presentes que significan la mortalidad y la morbilidad actuales. La reducción en la frecuencia y gravedad de las enfermedades que acosan a la población la fortalece y aumenta su resistencia y capacidad de concentración, lo que debería nutrir la productividad laboral directamente, e indirectamente por vía de una mayor capacidad de aprender e innovar. Además, bajas en la mortalidad y en la morbilidad permiten aprovechar mejor los recursos gastados en formar a las personas, al alargar el tiempo en que la sociedad puede cosechar los frutos de esas inversiones. La mayor duración de la vida activa y su menor interrupción por concepto de enfermedad, aumentan el numerador, y así el nivel, de la tasa de beneficio que reporta la inversión en personas. Gastos exitosos en salud deberían igualmente aminorar las pérdidas de recursos corrientes que significan las enfermedades y su curación. Los montos ahorrados podrían vertirse entonces en otras inversiones y en mejorar los niveles de consumo actuales. Por cierto, la magnitud del ahorro por los conceptos enunciados variará según la aflicción concreta y su frecuencia; los rendimientos puramente económicos de gastos en curación y preven- 
ción de enfermedades se han revelado o prometen ser altos en algunos casos y poco importantes en otros.

\section{Vi. Alternativas de salud}

La intervención colectiva que es la salud pública cobra su razón de existir de los efectos indirectos negativos, pero la orientación de sus programas concretos es resultado y barómetro de la pugna entre los diversos grupos de interés que forman la sociedad. Por ejemplo, en cuanto a las actividades no hogareñas y la insidiosa contaminación ambiental, cuyos daños a la salud no traslucen de inmediato, la presión colectiva por la internalización financiera de los costos previamente socializados puede derivar peligrosamente hacia lo más sacro de la gestión empresarial: el proceso de trabajo mismo, y la selección de productos y técnicas. Tanto la captación de recursos para suplir las necesidades materiales de la intervención colectiva en materia de salud, como el uso mismo de esos recursos, revisten dimensiones distributivas que calcan la estructura de poder vigente. La recaudación de fondos, por medio de diversos mecanismos tasativos, monetarios y de ingresos, y su asignación entre distintos programas de salud pública, favorecen más a ciertos grupos sociales que a la de otros. Basta con trazar la distribución del ingreso medio y de indicadores de salud dentro de una ciudad: la correlación espacial entre ambas será alta y positiva.

Las características de clase, o desigualdades distributivas, que posiblemente encierra una política de salud pueden ser ilustradas con los "perfiles de salud" de la gráfica 1, basados en que los gastos estatales y privados en salud se dirigen a la medicina curativa o a la preventiva y, en cada una de ellas, pueden proporcionar un servicio de calidad para una minoría ("servicio de élite") o un servicio masivo pero siempre de calidad.

El perfil de salud alinea, de izquierda a derecha, la fracción del gasto total en salud hecho por el fisco y particulares, absorbido por cada uno de los cuatro renglones: medicina curativa de élite, medicina preventiva de élite, medicina curativa masiva y medicina preventiva masiva. En la gráfica 1 , las fracciones son representadas cada una por una barra, de manera que la suma de las áreas de las barras sea igual a uno. Un perfil en forma de $J$ invertida (gráfica 1, panel $A$ ) reflejaría la situación actual en muchos países del Tercer Mundo, donde la medicina curativa de élite - con hospitales y personal altamente tecnificados - absorbe la parte del león de los recursos sociales encaminados a salud. Un perfil en forma de $J$ (gráfica 1, panel $B$ ) demuestra una asignación de esos recursos más justa y equitativa, en vista de los problemas de salud imperantes, con el grueso del esfuerzo dirigido al servicio masivo y el énfasis en la medicina preventiva - a través de clínicas y personal medianamente tecnificados y extensa difusión de información y acción colectiva contra los efectos indirectos negativos ya mencionados. Sería instructivo calcular perfiles de 


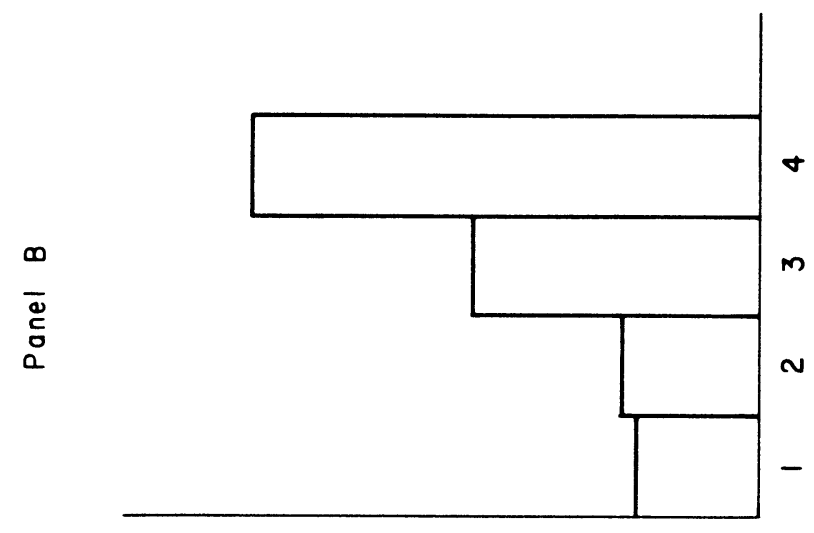

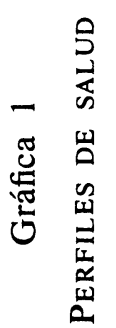

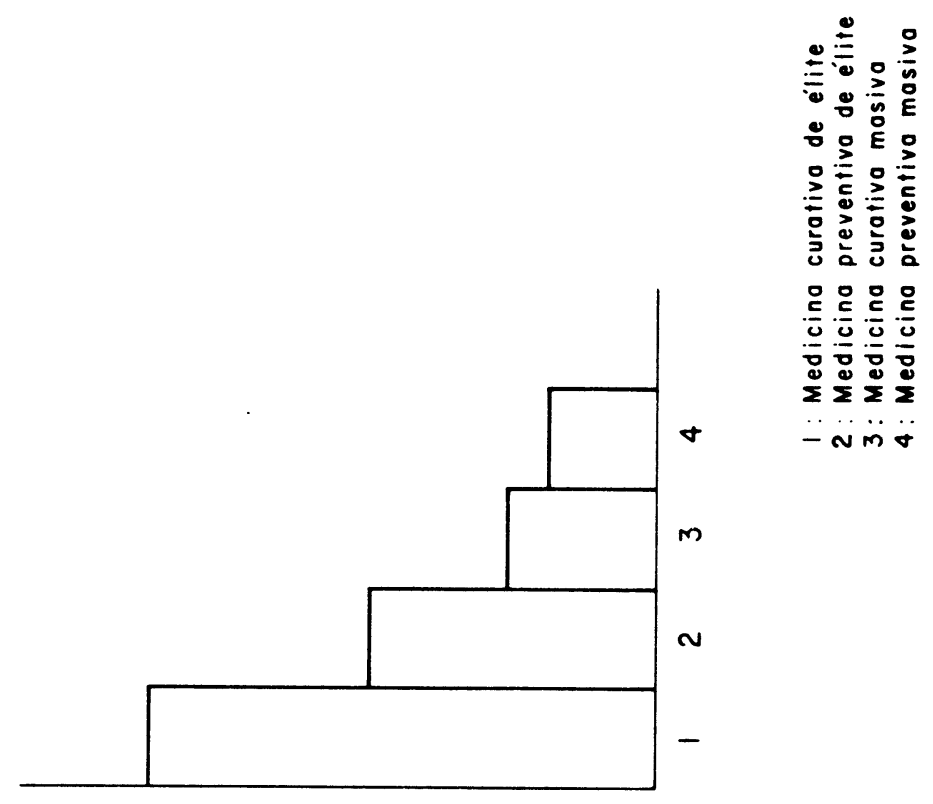

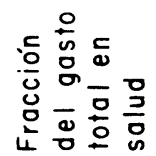


salud para el gasto privado y el gasto fiscal en salud por separado, con el fin de ver en qué medida el uno está remediando las carencias del otro. Por ejemplo, el gobierno puede tratar de suplir las deficiencias de una medicina curativa de élite, mantenida por una minoría que concentra en sus manos el producto social y elude en lo posible contribuir a la eliminación de los efectos indirectos negativos sobre la salud derivados de las diversas actividades sociales. Sin embargo, es ilusión esperar que la acción estatal, en una sociedad de desigual acceso al poder, supla de manera íntegra y sin conmociones políticas las deficiencias de un perfil de salud privado con forma de $J$-invertida, convirtiéndolo en un perfil social con forma de $J$. Esto por los costos políticos de tal acción.

Planificadores y otros científicos relacionados con problemas mundiales de salud concuerdan en que es la medicina preventiva masiva la que más falta hace en el Tercer Mundo. Esta medicina, más radical porque ataca las raíces del problema de salud, es inseparable de una política de aumentar el ingreso medio de los hogares pobres: esos hogares son focos de reproducción de enfermedad, tanto dentro de sí mismo como por sus consecuencias indirectas, las cuales, cabe añadir, las sufren principalmente otros hogares pobres, en virtud de que la segregación espacial se ocupa de separar los pobres de los ricos, los insalubres de los salubres.

Pero tal política de ingresos implica una redistribución del producto social, lo que a su vez significa alterar el poder de distintos grupos de presión sobre el proceso de trabajo social y los instrumentos de producción. El costo de un programa integral de salud como el aquí bosquejado, medido en términos de la pérdida de poder que significa para ciertos grupos de interés, no es fácil de cobrar. Mientras el costo político de un perfil social de salud equitativo, en forma de $J$, grava a los grupos de interés más pudientes, los costos políticos de un perfil de salud más desigual, en forma de $J$-invertida, grava a los estratos pobres bajo la forma de alta mortalidad y morbilidad.

Esta es la alternativa de salud a que se enfrentan los países del Tercer Mundo y que, como tantos otros problemas de trascendencia social, consiste en la pugna entre los intereses contradictorios de distintos grupos sociales. Vale añadir que la misma alternativa surge en muchos de los países ahora desarrollados, que albergan en su seno extensas capas de hogares económicamente desaventajados, donde se reproducen condiciones de salud como las que afligen a nuestros pueblos, y por las mismas razones. 